\title{
Análisis de la confiabilidad del sistema de molienda en una planta concentradora, basado en la criticidad
}

\begin{abstract}
RESUMEN
El objetivo del presente estudio es presentar el proceso de molienda en la planta concentradora de minerales de una empresa minera, describiendo las configuraciones de sus subprocesos y realizando el cálculo de la confiabilidad del sistema de molienda basado en la criticidad. Asimismo, se calculan los principales indicadores de mantenimiento por cada equipo del área de molienda. Los resultados facilitan a la empresa tomar decisiones de mantenimiento aplicando el monitoreo permanente de sus equipos de molienda, que son de uso cotidiano de los operarios, estableciendo la criticidad de cualquier equipo en general respecto a la ocurrencia de falla.

Palabras clave: criticidad, confiabilidad, mantenimiento
\end{abstract}

ANALYSIS OF SYSTEM RELIABILITY GRINDING IN A CONCENTRATOR, BASED ON THE CRITICALITY

\section{ABSTRACT}

\section{ABSTRACT}

The aim of this study is to present the process of grinding the ore concentrator plant a mining company, describing the settings of your threads and performing the calculation of the milling system reliability based on the criticality. Also, the main indicators are calculated for each team maintenance grinding area. The results enable companies maintenance decisions applying the constant monitoring of its milling equipment, which are of daily use of operators, establishing the criticality of any team in general regarding the occurrence of failure.

Keywords: criticality, maintenance, reliability

\section{INTRODUCCIÓN}

El estudio tiene por finalidad presentar los modelos utilizados en el proceso de molienda en la planta concentradora de minerales de una empresa minera y aplicar la simulación de diversos escenarios posibles para hallar la confiabilidad del sistema de molienda. Se determinan los indicadores de mantenimiento para los equipos de molienda del área de producción de la empresa.

La compañía es un complejo minero polimetálico ubicado en los Andes del Perú, que produce concentrados de cobre y zinc, y, como subproductos concentrados de molibdeno, plata y plomo. Para obtener los diferentes concentrados cuenta con una planta concentradora ubicada a más de

4,000 m.s.n.m., la cual procesa diversos tipos de minerales clasificados según los niveles de $\mathrm{Cu}, \mathrm{Zn}, \mathrm{Bi}$ y $\mathrm{Pb}$. En la siguiente tabla presentamos los 8 tipos de minerales o campañas que dispone:

Tabla 1. Tipos de Campañas de Cobre

\begin{tabular}{|c|}
\hline MINERALES DE COBRE \\
\hline M1 : Cobre bajo bismuto \\
\hline M2 : Cobre muy alto bismuto \\
\hline M3 $:$ Cobre alto bismuto \\
\hline M 4 : Cobre con bornita \\
\hline
\end{tabular}

Fuente: Elaboración propia.

Tabla 2. Tipos de Campaña de Zinc

\begin{tabular}{|l|}
\hline \multicolumn{1}{|c|}{ MINERALES DE ZINC } \\
\hline M3 : Cobre-Zinc bajo bismuto \\
\hline M4: Cobre -Zinc alto bismuto \\
\hline M2 : Cobre-Zinc muy alto bismuto \\
\hline M5 : Cobre-Zinc con bornita \\
\hline
\end{tabular}

Fuente: Elaboración propia.

* Master en OptimIzación. Profesora en la Facultad de Ciencias Matemáticas, Departamento de Investigación Operativa de la UNMSM. E-mail: esther berger_v@yahoo.es

** Master en Matemática. Profesor en la Facultad de Ciencias Matemáticas, Departamento de Matemática de la UNMSM. E-mail: lnuneztassarel27@yahoo.com

*** Master en Gerencia del Mantenimiento. Profesor en la Escuela de Posgrado de la Facultad de Ingeniería Mecánica-Energía de la Universidad Nacional del Callao. E-mail: anwaryarin@gmail.com 
Conocer la campaña permitirá tener una mejor gestión del mantenimiento, porque se logrará identificar qué equipos de la planta concentradora no se encuentran en funcionamiento, de esta manera se programará actividades correspondientes a su mantenimiento preventivo aprovechando la disponibilidad para su intervención.

\section{Procesos claves de la empresa minera y concentradora:}

Dentro del proceso de producción de concentrados de $\mathrm{Cu}$ y $\mathrm{Zn}$, se lleva a cabo el siguiente flujo de procesos:

Tabla 3. Procesos Claves de la empresa minera y concentradora

\begin{tabular}{|c|c|c|}
\hline $\begin{array}{l}\text { OPERACIÓN } \\
\text { en MINA }\end{array}$ & CONCENTRADO & $\begin{array}{l}\text { PUERTO } \\
\text { PUNTA } \\
\text { LOBITOS }\end{array}$ \\
\hline $\begin{array}{c}\text { Minado } \\
\text { Carguío y } \\
\text { acarreo } \\
\text { Chancadora } \\
\text { primaria } \\
\text { Traslado en faja } \\
\text { Apilamiento }\end{array}$ & $\begin{array}{c}\text { Molienda } \\
\text { Flotación } \\
\text { Presa de relaves } \\
\text { Minero } \\
\text { ducto }\end{array}$ & $\begin{array}{c}\text { Filtrado } \\
\text { Almacenamiento } \\
\text { en puerto } \\
\text { Embarque de } \\
\text { mineral }\end{array}$ \\
\hline
\end{tabular}

Fuente: Elaboración propia.

Figura 2.

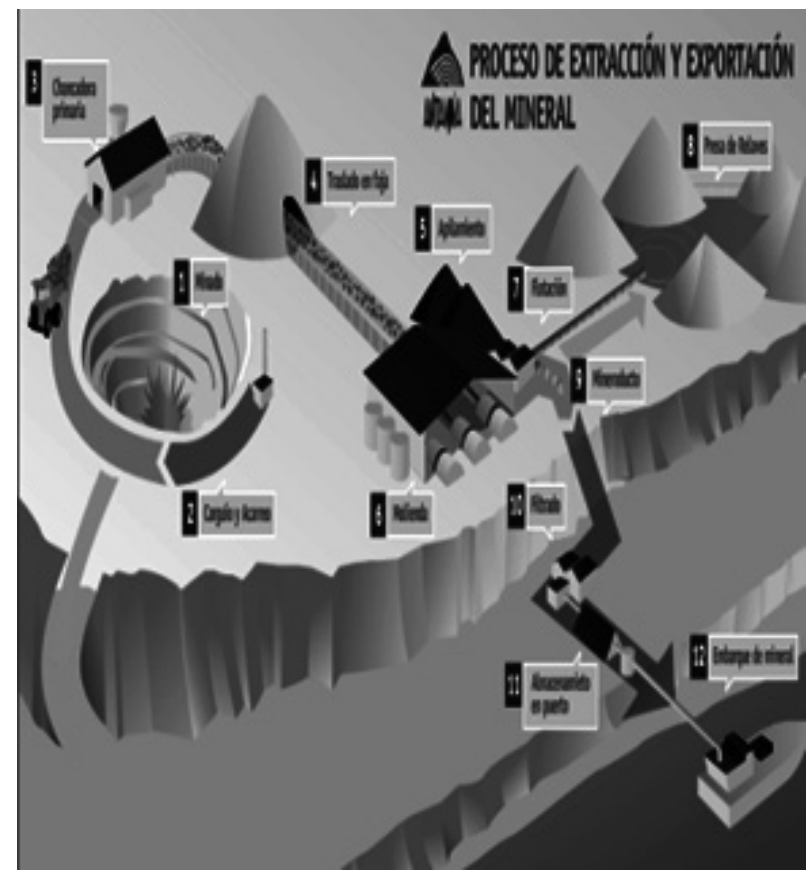

Fuente: Elaboración propia. Proceso de Extracción y Exportación.
Figura 2. Principales Procesos Claves

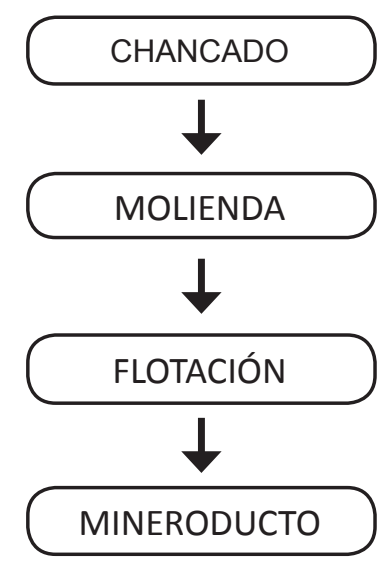

Fuente: Elaboración propia

\section{PROCESO DE MOLIENDA}

\section{DESCRIPCIÓN DEL PROCESO DE MOLIENDA}

A. Zona de Acumulación de Material

Se cuenta con 2 Stackers :

El primer Stacker tiene la capacidad de formar 2 pilas:

Pila A (Bajo Bismuto-Cu): campañas M1-M3-M5-M6

Pila B (Alto Bismuto-Zn): campañas M2-M2A, M4,M4A

El segundo Stacker:

Sólo forma pila C, así, esta pila abarca a cualquier campaña.

Las pilas A y B alimentan a la faja CVB-004 a través de los apronfeeder :

Pila A: Utiliza los apronfeeder FEA-10, FEA-11, FEA-12.

Pila B: Utiliza los apronfeeder FEA-13, FEA-14, FEA-15.

Las pilas C alimentan a la faja CVB-626 a través de los apronfeeder :

Pila C: Utiliza los apronfeeder FEA-35, FEA-36, FEA-37.

\section{B. Circuito SAG 01}

La faja CVB-004 alimenta directamente al molino SAG 01. Se realiza la molienda a través de bolas de acero de 5 pulgadas ingresadas en el molino, también se suministra agua y reactivos para acondicionar el material para la fase de flotación (PAX-Cianuro-Cal, entre otros).

Realizada la molienda en el SAG 01 se envía el material al cajón SUL-013, este cajón cuenta con 2 bombas (Por confiabilidad solo 1 trabaja, la otra es de redundancia o Stand By) esta bomba envía la pulpa al distribuidor radial que corresponda: 
PPS $609 \rightarrow$ Envía al distribuidor STP 673

PPS $602 \rightarrow$ Envía al distribuidor STP 674

\section{Circuito SAG 02}

La faja CVB-626 alimenta a la faja CVB 628 esta alimenta directamente al SAG 2, se utiliza el mismo mecanismo de molienda que el SAG 01 y se envía la pulpa al SUL-014, este cajón también cuenta con 2 bombas (por confiabilidad sólo una trabaja siendo la otra de redundancia o stand by). Esta bomba envía la pulpa al distribuidor radial que corresponda:

PPS $600 \rightarrow$ Envia al distribuidor STP 673

PPS $601 \rightarrow$ Envia al distribuidor STP 674

\section{Distribuidores Radiales}

Los distribuidores radiales son dos: STP 673 y STP 674 (por confiabilidad sólo uno trabaja, la otra es de redundancia o Stand By). Este distribuidor radial envía a los cuatro cajones SUL que alimentarán a los cuatro nidos de ciclones (cada nido de ciclón cuenta con 13 ciclones).

La distribución es la siguiente:

Cajón SUL-01 $\rightarrow$ utiliza PPS-612 para alimentar al nido de ciclones 1
Cajón SUL-02 $\rightarrow$ Utiliza PPS-613 para alimentar al nido de ciclones 2 Cajón SUL-03 $\rightarrow$ Utiliza PPS-614 para alimentar al nido de ciclones 3

Cajón SUL-04 $\rightarrow$ Utiliza PPS-611 para alimentar al nido de ciclones 4

\section{E. Nido de Ciclones}

La función del nido de ciclones es clasificar el material. Material fino: Es enviado a la zona de flotación.

Material grueso: Es ingresado al molino de bolas correspondiente.

EL molino de bolas realiza el proceso de molienda con bolas de acero de 2 y 3 pulgadas de diámetro para obtener una menor granometría, terminado el proceso de molienda se envía nuevamente al SUL respectivo:

Molino de Bolas MLB $001 \rightarrow$ SUL 001

Molino de Bolas MLB $002 \rightarrow$ SUL 002

Molino de Bolas MLB $003 \rightarrow$ SUL 003

Molino de Bolas MLB $004 \rightarrow$ SUL 004

Así, nuevamente las bombas envían la pulpa a los nidos de ciclones para su clasificación, realizándose el proceso en lazo cerrado.

Figura 3. Proceso de molienda 1.

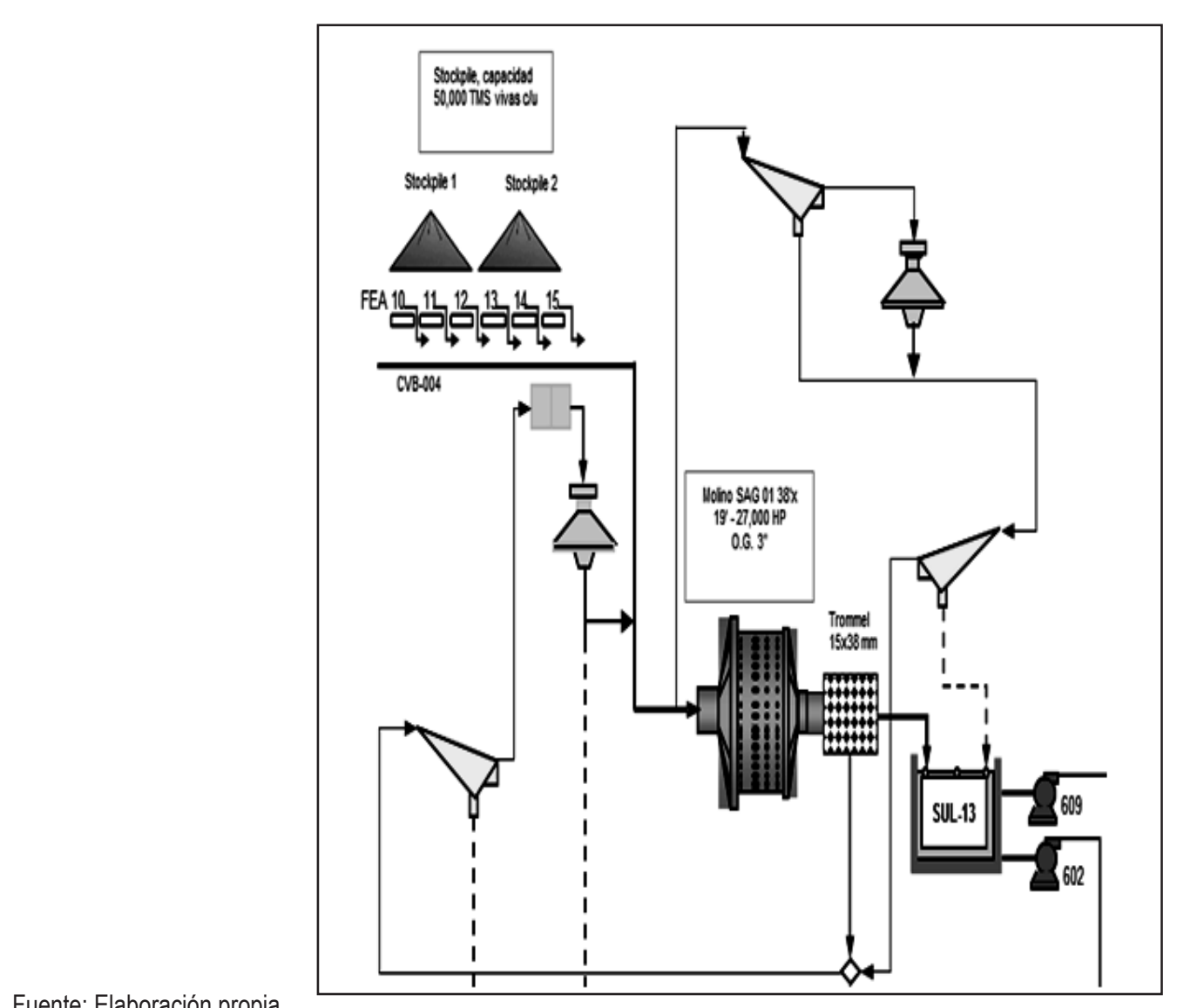


Figura 4. Proceso de molienda 2.

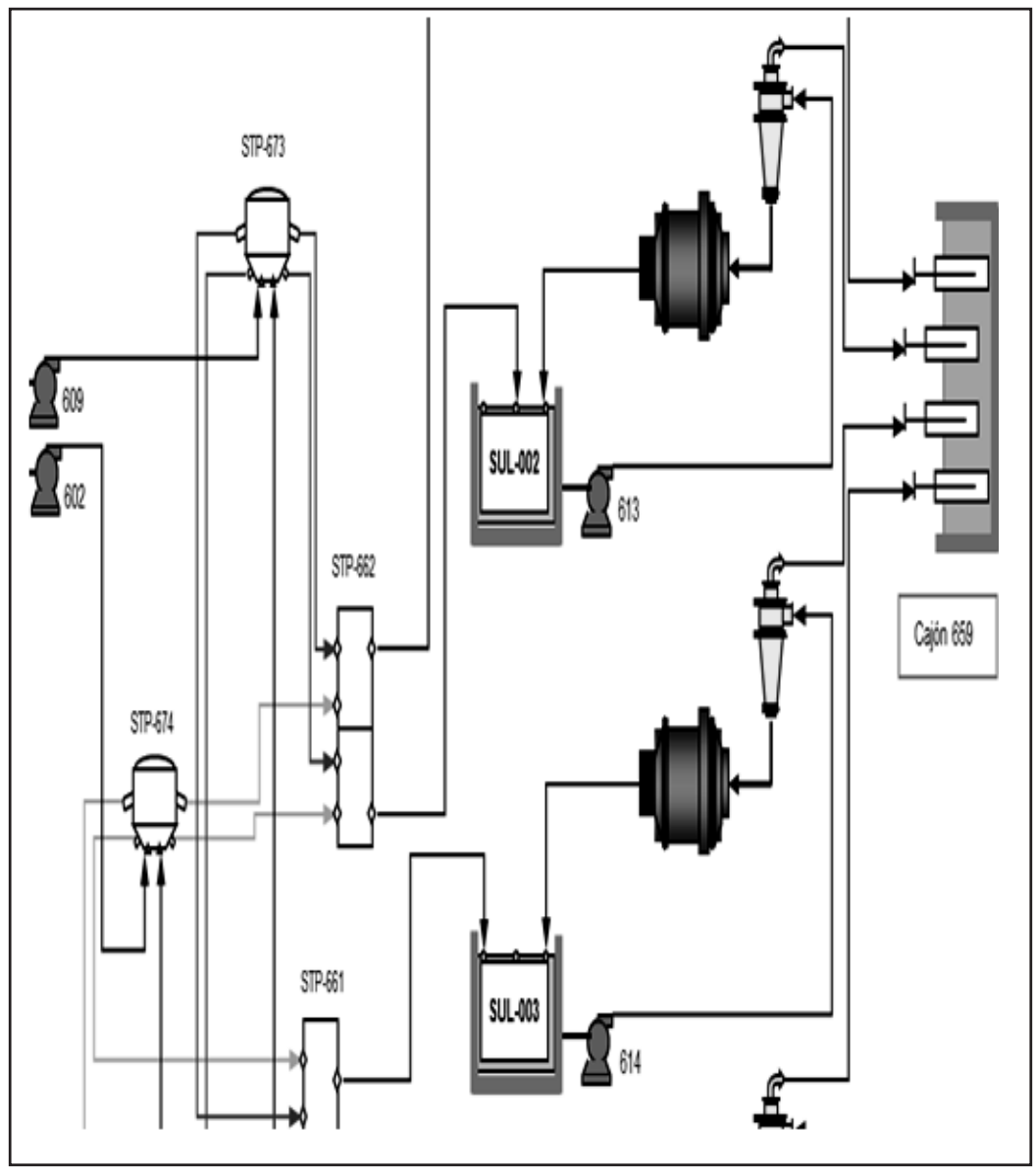

Fuente: Elaboración propia.

\section{PROBLEMA}

No existe en la empresa un proceso que permita identificar y monitorear permanentemente la confiabilidad del sistema de molienda y desarrollar un sistema eficiente de mantenimiento planificado o basado en la confiabilidad.

Por esta razón se plantea lograr los objetivos de:

- Establecer un proceso de identificación y monitoreo permanente de la confiabilidad del sistema de molienda.

- Utilizar el mantenimiento basado en la confiabilidad aplicado al sistema de molienda de la planta concentradora.

- Analizar los resultados del enfoque del mantenimiento basado en la confiabilidad y revisar las ventajas y desventajas que se puedan presentar en su proceso de implementación.

Asimismo, se desea alcanzar la meta de utilizar las herramientas del mantenimiento basado en la confiabilidad como sistema de gestión del mantenimiento en la planta productora y concentradora.

Unresultadodelestudio proporcionaunaherramienta de cálculo de los principales indicadores de mantenimiento, que permite definir oportunidades de mejora para elevar el nivel de desempeño y eficiencia del área de mantenimiento de la empresa. Se da el primer paso para complementar y mejorar el estudio de la confiabilidad de todos los procesos productivos claves de la empresa.

Asimismo, como resultado del estudio es posible identificar:

- La criticidad de cada equipo del sistema de molienda.

- Hallar el nivel de confiabilidad de cada equipo, basado en el análisis de criticidad.

- Por ser el primer trabajo, representa un piloto para futuros trabajos de análisis de confiabilidad basado en criticidad. 
Los beneficiarios son:

- Operaciones Concentradora, que tras una gestión basada en la confiabilidad espera aumentar la disponibilidad de todos los equipos críticos de molienda.

- Mantenimiento Concentradora, con la optimización de las tareas de mantenimiento, proyectándose a hacer más eficientes sus actividades de mantenimiento preventivo y correctivo.

- La empresa, que con el mejoramiento de los niveles de disponibilidad se proyecta hacia una mayor producción y seguridad en las operaciones asociadas a mayores utilidades.

\section{MARCO TEÓRICO}

\section{Mantenimiento}

El mantenimiento es un componente importante del proceso de calidad de los productos y una estrategia para ejercer la competencia con éxito. Es un sistema paralelo y estrechamente ligado al sistema de producción.

Un sistema de producción se considera como un medio que convierte insumos como materias primas y mano de obra, en productos terminados que satisfacen las necesidades de los clientes, pero una salida secundaria es la falla de un equipo que genera una demanda de mantenimiento.

El sistema de mantenimiento toma esa demanda como entrada, agrega conocimiento especializado, mano de obra, reparación y produce como salida un equipo que funciona correctamente y que tiene una capacidad de producción.

Mientras que un sistema de producción tiene como objetivos maximizar las utilidades satisfaciendo la demanda del mercado, con la más alta calidad y oportunidad, un sistema de mantenimiento contribuye a alcanzarlos objetivos minimizando los tiempos muertos, mejorando la calidad, aumentando la productividad y la eficacia en el cumplimiento oportuno de los pedidos a los clientes.

\section{Estrategias de Mantenimiento}

Respecto al mantenimiento de equipos, existen diversas estrategias que se aplican individualmente o combinadas: Boreo (2009) [2]

\section{Mantenimiento correctivo, de reparación o por fallas}

Se realiza cuando el equipo ha interrumpido su operación por haber perdido su capacidad de trabajo debido a una falla. Es un mantenimiento no planificado que ocurre cuando no se justifica o no es posible asumir el costo adicional del mantenimiento planificado. Ocurre en los equipos con componentes electrónicos o cuando no se dispone de recursos para remplazo o para mantenimiento preventivo.

\section{Mantenimiento Planificado o Basado en la Confiabilidad, Smith (2007) [5]; Duffua (2009) [3], que comprende:}

Mantenimiento preventivo basado en el tiempo o en el uso

Es un mantenimiento planificado para evitar o minimizar la posibilidad de falla en un equipo, con base en el tiempo o en un calendario de mantenimiento, 0 , se puede basar en el uso del equipo en un horizonte de tiempo.

\section{Mantenimiento Predictivo o Mantenimiento Preventivo Basado en las Condiciones}

Es un mantenimiento planificado que se realiza con base en las condiciones del equipo, determinadas mediante la observación de sus factores fundamentales a los que la condición de este afecta.

\section{Mantenimiento de Oportunidad}

Se realiza cuando se presenta un hecho que permite realizar un mantenimiento no planificado, por ejemplo, un paro general planificado en la planta de producción para mantenimiento de la planta, un paro fortuito por alguna razón laboral o por falta de energía.

Inspección para la detección de fallas

Se realiza para evaluar la posibilidad de existencia inicial de fallas.

\section{Modificación del Diseño}

Se modifica el diseño de un equipo para que alcance un estado adecuado de funcionamiento, implicando su mejora o la expansión de su capacidad.

\section{Reparación General}

Es la inspección detallada de todo el equipo y el restablecimiento del funcionamiento de todos sus elementos.

\section{Reemplazo}

El reemplazo puede ser planificado de acuerdo a una programación, es una estrategia que se aplica como resultado de una evaluación cuyo resultado indica que es más conveniente desde el punto de vista de los costos, de la calidad de los productos o de la obsolescencia tecnológica, reemplazar que hacer mantenimiento. 
Las diferentes estrategias no son excluyentes, la combinación adecuada de estas permite desarrollar una política de mantenimiento óptima para la empresa.

\section{Mantenimiento orientado a la Confiabilidad}

Surgió con el objetivo de analizar la eficacia de las reparaciones generales de componentes complejos de los aviones, las cuales se basaban en el tiempo para verificar si se reducía la frecuencia y el costo por fallas. Se llegó a la conclusión que esas reparaciones no afectaban la frecuencia de fallas.

En los equipos complejos la máxima probabilidad condicional de falla ocurre en la edad temprana del equipo, luego, baja a una tasa constante de falla y al final de la vida útil del equipo se incrementa.

Se asume que una reparación general vuelve a cero la edad del equipo y que por lo tanto incrementa la probabilidad de falla.

Una falla está relacionada con eventos aleatorios que aceleran el deterioro del equipo, tales como: sobre voltajes, incorrecta lubricación, operación incorrecta.

En este caso, el mantenimiento preventivo basado en las condiciones es útil para hacer un seguimiento del funcionamiento del equipo.

El mantenimiento orientado a la confiabilidad analiza el tipo de falla, el efecto que produce la falla y su nivel de gravedad.

\section{Proceso de mantenimiento orientado a la confiabilidad}

1. Determinar cuáles son los subsistemas más importantes del equipo.

2. Definir la función esperada del equipo y que tipo funcional de falla puede tener.

\section{Especificar las causas de la falla.}

4. Especificar los efectos de la falla en una secuencia de eventos: producción, seguridad, ambiente.

5. Determinar el nivel de gravedad de la falla.

6. Usar el tipo de mantenimiento adecuado.

7. Determinar la acción de prevención de falla y su frecuencia de aplicación con base en análisis histórico.

8. Si no existe una acción preventiva, analizar si puede seguir operando hasta que falle.

9. Rediseñar en caso necesario.

\section{Confiabilidad de Sistemas}

Es la probabilidad que un sistema cumpla con sus funciones sin fallar, durante un tiempo determinado. Facilita el conocimiento de la relación entre el tiempo de uso del sistema y la pérdida de su capacidad de funcionamiento por el surgimiento de alguna avería que puede producirse al inicio del uso del equipo por problemas en su fabricación o durante su etapa de plena actividad por causas propias de la actividad del equipo o durante la decadencia del equipo por su antigüedad, desgaste u obsolescencia.

\section{METODOLOGÍA}

Presentamos en modo piloto la metodología de análisis del sistema molienda que se inicia con el Análisis de Criticidad aplicado a los equipos que pertenecen al sistema en mención, teniendo en cuenta lo siguiente: (Moubray (2004) [6].

$$
\begin{gathered}
\text { CRITICIDAD }=\text { Frecuencia de Falla * } \\
\text { Consecuencia }
\end{gathered}
$$

Donde:

Consecuencia $=(($ Nivel de producción $x$ TMPR $x$ Imp. Producción) + Costo de reparación + Impacto en seguridad + Impacto ambiental)

Figura 4. Tipos de criticidad

\begin{tabular}{|c|}
\hline CRÍTICO \\
\hline SEMICRÍTICO \\
\hline NO CRÍTICO \\
\hline
\end{tabular}

Fuente: Elaboración propia.

Luego se debe elaborar el Análisis de Modos y Efectos de Fallas (AMEF) basándonos en el diagnóstico de criticidad, para nuestro caso en estudio los motores del sistema de molienda resultaron ser los más críticos.

En el Análisis de Modos y Efectos de Fallas se realiza un desagregado de los componentes de los motores, los cuales se detallan a continuación: estator, rotor y sistemas auxiliares. Amendola (2002) [1].

Asimismo para este análisis se ha tenido en cuenta los siguientes aspectos:

- Efecto sobre el servicio.

- Valor Técnico Económico.

- Flexibilidad del equipo en el sistema. 
- Dependencia Logística.

- Efecto de la falla.

Cabe mencionar que las ponderaciones consideradas en el Análisis de Criticidad de
Equipos están basadas en los criterios de expertos los cuales han realizado consultorías similares en diversas empresas del rubro minero. En este caso en particular se trabajó con la ponderación que se detalla a continuación:

Tabla 4. Ponderaciones consideradas en el Análisis de Criticidad

\begin{tabular}{|c|c|c|}
\hline \multicolumn{3}{|c|}{ ISOACTO EN SEGURIOAO } \\
\hline $\mathrm{N}^{*}$ & GIIRO & PulkTAL \\
\hline 0 & 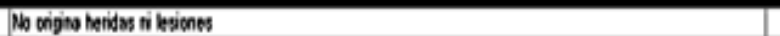 & 0 \\
\hline 1 & 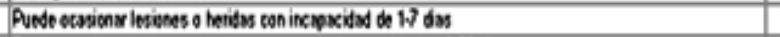 & 2 \\
\hline 2 & 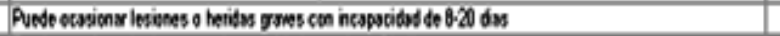 & 4 \\
\hline 9 & 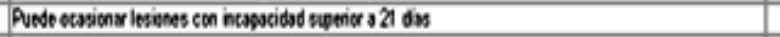 & 6 \\
\hline 4 & 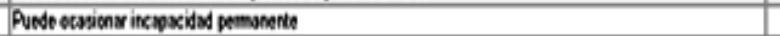 & 8 \\
\hline 5 & Muete & 10 \\
\hline \multicolumn{3}{|c|}{ 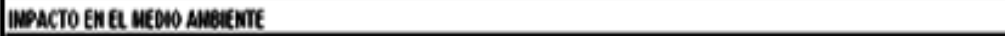 } \\
\hline $\mathrm{n}^{*}$ & CAll kno & Puliant \\
\hline 1 & 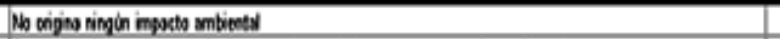 & 0 \\
\hline 2 & 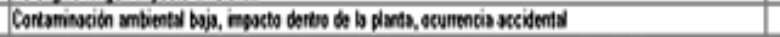 & 1 \\
\hline 3 & 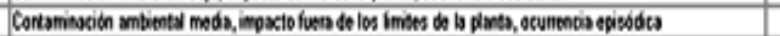 & 2 \\
\hline 4 & 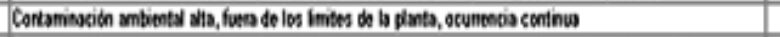 & 3 \\
\hline 5 & 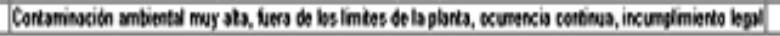 & 4 \\
\hline \multicolumn{3}{|c|}{ 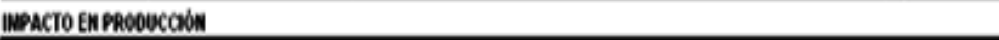 } \\
\hline $\mathrm{n}^{*}$ & (\$IIIHO & Pukiant \\
\hline 1 & Tho dicts la producotin & 0 \\
\hline 2 & 18. 25s de impats & 2 \\
\hline 3 & $20 x \cdot 605$ on mpoto & 4 \\
\hline 4 & SIN-75s do mpoto & 6 \\
\hline 5 & 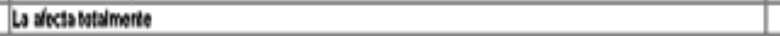 & 8 \\
\hline \multicolumn{3}{|c|}{ 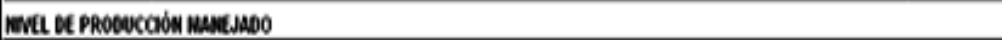 } \\
\hline$n^{*}$ & C에1 No & PUKIATL \\
\hline 1 & 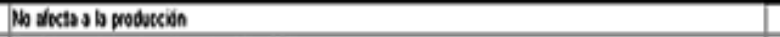 & 0 \\
\hline 2 & Pordden en la production mite Is. $25 x$ & 1 \\
\hline 3 & 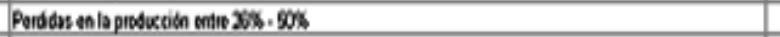 & 2 \\
\hline 4 & 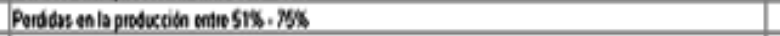 & 3 \\
\hline 5 & 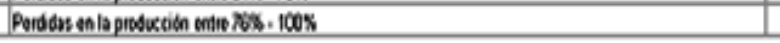 & 4 \\
\hline \multicolumn{3}{|c|}{ 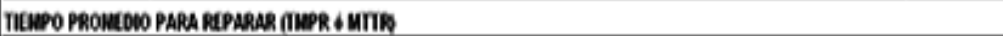 } \\
\hline $\mathrm{N}^{*}$ & C제I NO & Puktalt \\
\hline 0 & Monot a 20minutos & 1 \\
\hline 1 & Entio 20 minutses y hats & 2 \\
\hline 2 & Enter I toe y 3 how & 3 \\
\hline $\mathbf{3}$ & Entre J then y 0 then & 4 \\
\hline 4 & Entle d bes y 16 has & 5 \\
\hline 5 & Entre 16 hasas a mas & 6 \\
\hline \multicolumn{3}{|c|}{ FRECUEMCA DE FALLA } \\
\hline$n^{*}$ & CAll Hio & PUKTAL \\
\hline 0 & 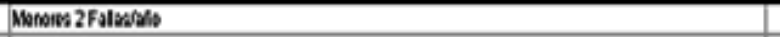 & 1 \\
\hline 1 & Entele 24 Falowido & 2 \\
\hline 2 & 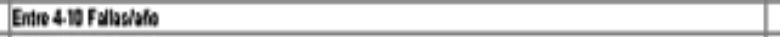 & 3 \\
\hline 3 & Enter 1030 Falateds & 4 \\
\hline 4 & Emite 3050 Falesols & 5 \\
\hline 5 & Mayoum a SOF Falzado & 6 \\
\hline \multicolumn{3}{|c|}{ COSTOS DE REPARACIÓn } \\
\hline$N^{*}$ & CAl1 HO & PUTTAN \\
\hline 1 & Momen atsi.2000 & 1 \\
\hline 2 & Entre SC. 2000 y SC. 2000 & 2 \\
\hline 3 & Entio S. 000 y 8.2000 & 3 \\
\hline 4 & Entre SL. 8000 y S. 10000 & 4 \\
\hline 5 & Mayor as: 18000 & 5 \\
\hline
\end{tabular}

Fuente: Elaboración propia. 
Tabla 5. Análisis de Criticidad de Equipos de la Empresa

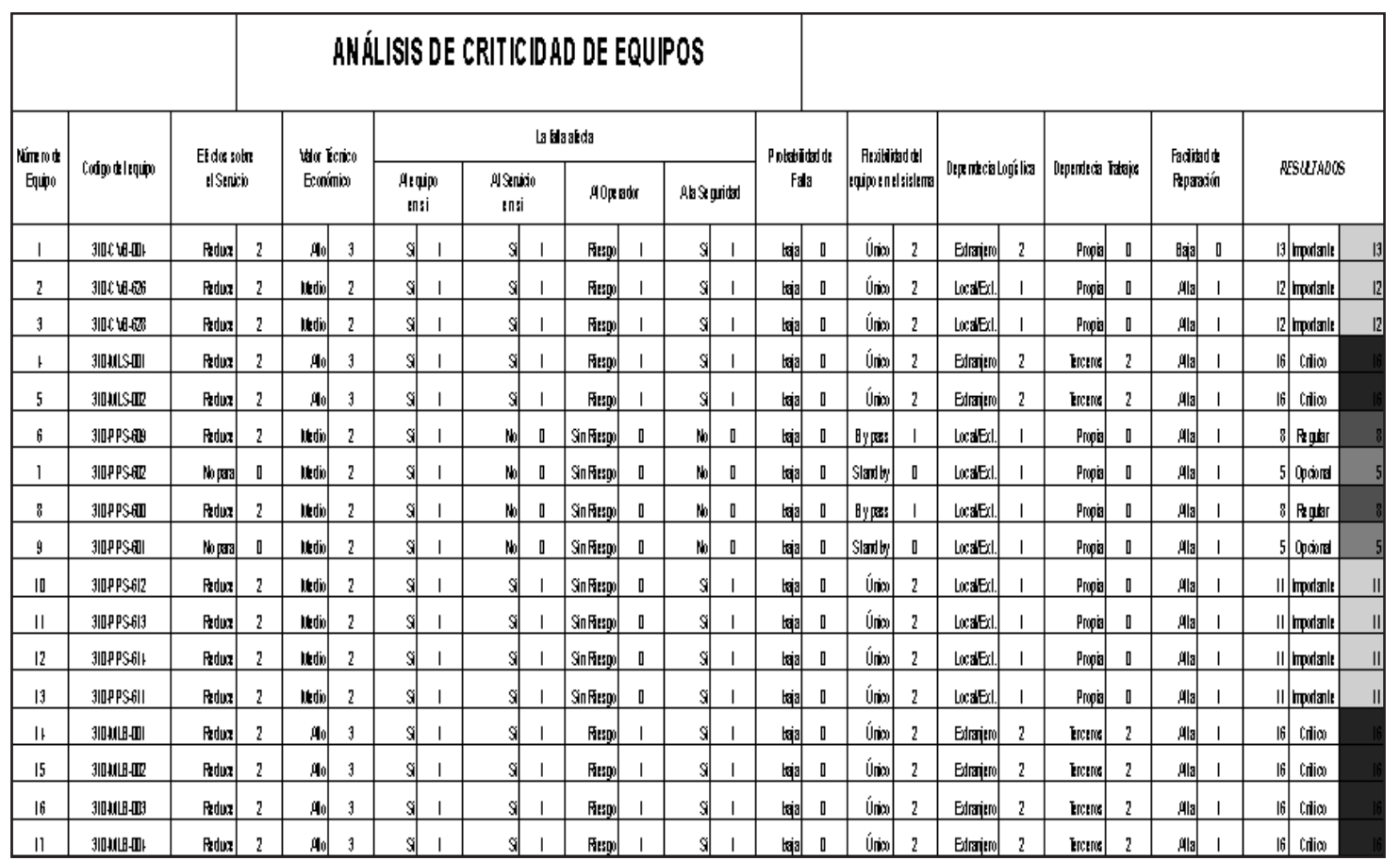

Fuente: Elaboración propia.

Tabla 6. Análisis del Modo de Falla del Estator

\begin{tabular}{|c|c|c|c|c|c|c|c|c|}
\hline & & \multicolumn{7}{|c|}{ ANÁLISIS DE MODO FALLA DE LOS MOTORES } \\
\hline \multicolumn{2}{|c|}{ 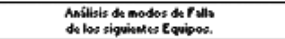 } & 310.MLs.001 & 310-Mts.002 & 300-MLB-001 & 310.Mer-002 & 300-med-000 & 310.MB. 004 & \\
\hline COMPONENTE & UB.COMPONENI & FALLA POTENCIAL & MODODEFALLA & FALLAA FUNCIONALL & EFECTOS DE LA FALLA & TAREA PREDICTIVA & \multicolumn{2}{|c|}{ CNOLOGIA PAEDICTRFAECUENCI } \\
\hline \multirow{7}{*}{ Estator } & \multirow{3}{*}{ 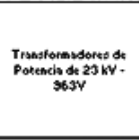 } & \multirow{3}{*}{ 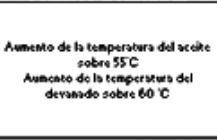 } & \multirow{3}{*}{ 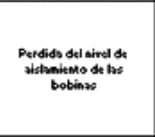 } & \multirow{3}{*}{ 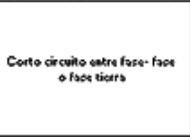 } & 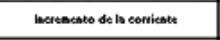 & 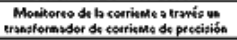 & 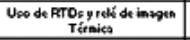 & comimos \\
\hline & & & & & 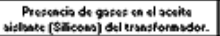 & 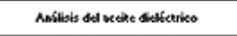 & 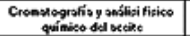 & tometrut \\
\hline & & & & & 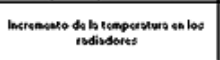 & 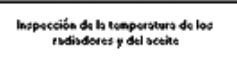 & 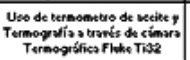 & Trimetaral \\
\hline & \multirow{2}{*}{ 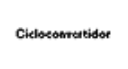 } & \multirow{2}{*}{ 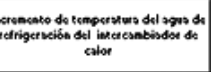 } & \multirow{2}{*}{ 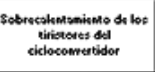 } & \multirow{2}{*}{ 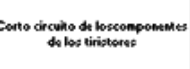 } & 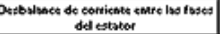 & 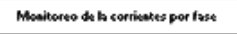 & 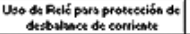 & Cominus \\
\hline & & & & & 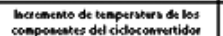 & 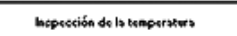 & 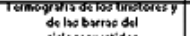 & semestrat \\
\hline & \multirow{2}{*}{ Botinso del estotor } & \multirow{2}{*}{ 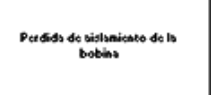 } & \multirow{2}{*}{ 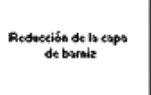 } & \multirow{2}{*}{ 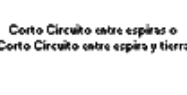 } & 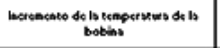 & 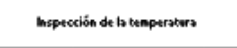 & 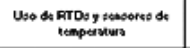 & Comineso \\
\hline & & & & & 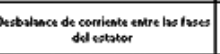 & 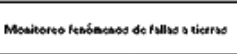 & 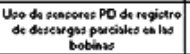 & Comimos \\
\hline
\end{tabular}

Fuente: Elaboración propia. 
Tabla 7. Análisis del Modo de Falla del Rotor y Sistemas Auxiliares

\begin{tabular}{|c|c|c|c|c|c|c|c|c|}
\hline \multirow{7}{*}{ Rotor } & \multirow{3}{*}{ 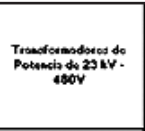 } & \multirow{3}{*}{ 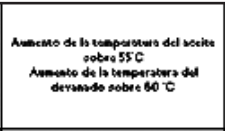 } & \multirow{3}{*}{ 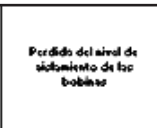 } & \multirow{3}{*}{ 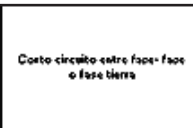 } & 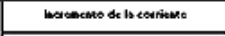 & 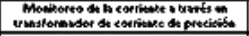 & 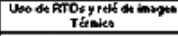 & 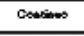 \\
\hline & & & & & 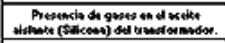 & 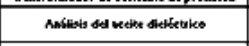 & 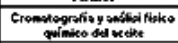 & Sentsosut \\
\hline & & & & & 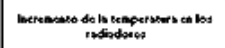 & 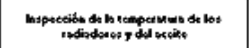 & 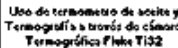 & Tiliessused \\
\hline & \multirow{2}{*}{ Penditionder AC-DC } & \multirow{2}{*}{ 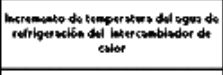 } & \multirow{2}{*}{ 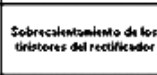 } & \multirow{2}{*}{ 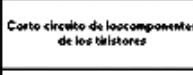 } & 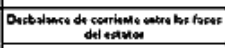 & 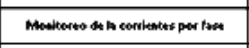 & 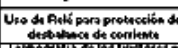 & cortinen \\
\hline & & & & & 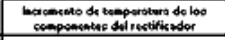 & 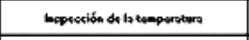 & 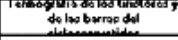 & samentad \\
\hline & \multirow{2}{*}{ 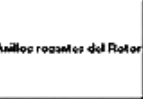 } & \multirow{2}{*}{ 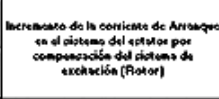 } & \multirow{2}{*}{ 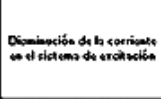 } & \multirow{2}{*}{ 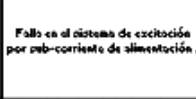 } & 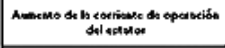 & 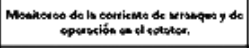 & 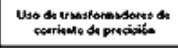 & conthes \\
\hline & & & & & 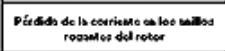 & 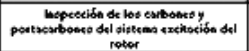 & 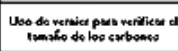 & extrineteral \\
\hline \multirow{7}{*}{$\begin{array}{l}\text { SISTEMAS } \\
\text { AUXILIARES }\end{array}$} & \multirow{3}{*}{ Sibetens de ceftigascition } & \multirow{3}{*}{ Butolition as ate } & \multirow{3}{*}{ 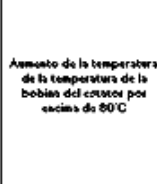 } & \multirow{3}{*}{ 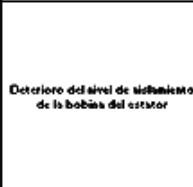 } & 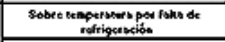 & 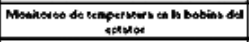 & 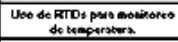 & contsons \\
\hline & & & & & 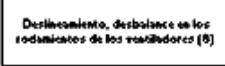 & 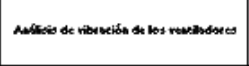 & 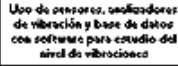 & consinsos \\
\hline & & & & & 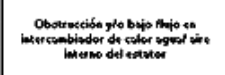 & 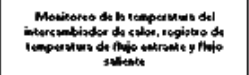 & 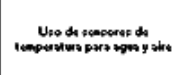 & conthon \\
\hline & \multirow{2}{*}{ 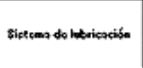 } & \multirow{2}{*}{ 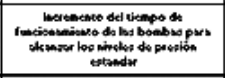 } & \multirow{2}{*}{ 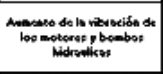 } & \multirow{2}{*}{ 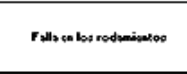 } & 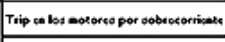 & 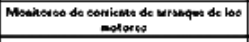 & 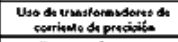 & contions \\
\hline & & & & & 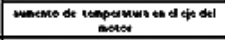 & 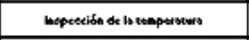 & 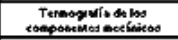 & stanstod \\
\hline & \multirow{2}{*}{ 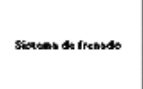 } & \multirow{2}{*}{ 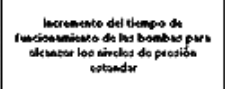 } & \multirow{2}{*}{ 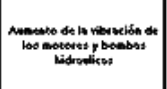 } & \multirow{2}{*}{ 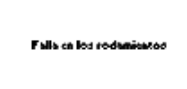 } & 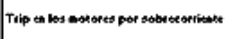 & 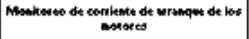 & 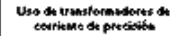 & cosises \\
\hline & & & & & 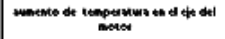 & 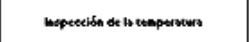 & 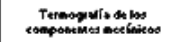 & scanstsod \\
\hline
\end{tabular}

Fuente: Elaboración propia.

\section{CONCLUSIONES}

1. Como resultado del Análisis de Criticidad de los Equipos se obtiene que los motores del sistema de molienda son los más críticos.

2. Utilizando el AMEF ha sido posible elaborar una herramienta para la operación y monitoreo de los motores del sistema de molienda, los cuales son usados por los operarios en sus labores cotidianas.

\section{REFERENCIAS BIBLIOGRÁFICAS}

[1] AMÉNDOLA, L. (2002). Modelos Mixtos de Confiabilidad. DATASTREAM. España: Valencia.
[2] BOREO Carlos. (2009). Mantenimiento Industrial. Ed. Universitas. Córdoba.

[3] DUFFUA Salih; RAOUF A.; DIXON C. John. (2009). Sistemas de Mantenimiento, Planeación y Control. Limusa-Wisley. México.

[4] MOUBRAY, J. (2004). Mantenimiento Centrado en Confiabilidad. RCM II. USA: Lillington.

[5] SMITH, A. (2007). Reliability Centered Maintenance, McGraw Hill. N.Y. 